\title{
Dialysis Catheter Exit Site Infection
}

National Cancer Institute

\section{Source}

National Cancer Institute. Dialysis Catheter Exit Site Infection. NCI Thesaurus. Code

C114727.

Local infection at the dialysis catheter exit site. 